\title{
ANAM vs. NAM: Is the Difference Significant?
}

\author{
Steve Hammel ${ }^{a}$, Alexander van $\operatorname{Eijk}^{b}$, Dimitri Tsintikidis ${ }^{a}$, \\ ${ }^{a}$ Space and Naval Warfare Systems Center, San Diego CA \\ ${ }^{b}$ TNO Defence, Security and Safety, The Hague, Netherlands
}

\begin{abstract}
The Navy Aerosol Model (NAM, available in MODTRAN) is widely used as a tool to assess the aerosol extinction in the marine atmospheric surface layer. NAM was built as a regression model in the 1980s to represent the aerosol extinction at deck height as a function of the meteorological conditions. The recently developed Advanced Navy Aerosol Model (ANAM) utilizes additional experimental evidence to supersede NAM by correcting the underestimation of the concentration of aerosols larger than a few microns. More importantly, ANAM provides the aerosol extinction as a function of height between the surface and several tens of meters.

Present-day naval surveillance and threat scenarios require detection of targets at the horizon, such as seaskimming missiles, or small targets such as rubber boats. In either case, the propagation path from sensor to target is likely to come very close to the wave surface and in order to estimate detection ranges, an assessment of the transmission losses along the path is necessary.

To answer the question posed in the title, we assess the two models using two meteorological data sets (784 cases) representative of diverse maritime conditions in regions of interest around the world.
\end{abstract}

\section{INTRODUCTION}

Electro-optical (EO) systems have advanced significantly since the first sensors were conceived. However, the expected performance of modern surveillance systems or seeker heads is often not achieved in the field. Atmospheric conditions may seriously degrade sensor performance and thus become the determining factor for system performance. One of the factors limiting sensor performance is the scattering and absorption of optical radiation by atmospheric aerosols. The interaction of radiation and aerosols leads to transmission losses and hence to a reduction of the effective detection range against specific threats. For imaging systems, aerosol absorption and scattering result in a loss of contrast, and hence, image degradation, which makes it more difficult to classify and identify threats.

Aerosol extinction is the physical parameter that quantifies the aerosol absorption and scattering effects on sensor performance. The extinction $\alpha_{\text {aer }}(\lambda)$ specifies the total loss of EO-radiation of wavelength $\lambda$ per unit distance, and is commonly reported in $\mathrm{km}^{-1}$. The transmission $T$ through the atmosphere of EO-radiation over a particular distance $\mathrm{R}$ is then found by:

$$
T(R, \lambda)=\frac{I(R, \lambda)}{I_{0}(\lambda)}=\exp \left[-\alpha_{\mathrm{aer}}(\lambda) R\right]
$$

where $I_{0}$ denotes the intensity of the EO-radiation at the source and $I(R)$ represents intensity after propagating a distance $R$. The aerosol extinction can be evaluated by Mie theory if the aerosol particle size distribution (PSD, or number of aerosols present in the atmosphere as function of aerosol diameter D) and the chemical composition of the (individual) aerosol particles are known. Unfortunately, these two quantities are difficult to measure and even worse, they vary considerably in space and time.

In most naval applications, marine or sea-salt aerosols constitute a fair percentage of the total atmospheric aerosol loading. Sea spray is generated predominantly by the action of the wind on the ocean surface. Two mechanisms have been identified: bubble-mediated production of jet droplets from breaking waves ${ }^{1-3}$ effective at wind speeds from $4 \mathrm{~m} / \mathrm{s}$ and up, and production of spume droplets torn directly from wave crests by strong

Further author information: (Send correspondence to S.H.)

S.D.-H.: E-mail: stephen.doss-hammel@navy.mil, Telephone: 6195534578

Atmospheric Optical Modeling, Measurement, and Simulation, edited by Stephen M. Doss-Hammel, Anton Kohnle, Proc. of SPIE Vol. 5891, 58910G, (2005) · 0277-786X/05/\$15 · doi: 10.1117/12.618931 
turbulence $^{4}$ effective at wind speeds in excess of 10-12 m/s. Several details of the production mechanisms of sea spray are poorly understood, which makes it difficult to model precisely the amount of droplets that is generated from the surface (the so-called source function). In addition, measurements of the aerosol concentration very close to the surface are difficult to make, even in the laboratory under controlled conditions. As a result, the source function is not very well known and estimates vary widely as shown by Andreas. ${ }^{5}$

After their generation, the saline droplets find themselves in the turbulent airflow close to the surface. This flow is highly fluctuating due to the presence of the waves and necessitates a detailed and elaborate description. The turbulent airflow above waves has been the subject of numerous studies, starting with Miles ${ }^{6}$ (and see Belcher and $\mathrm{Hunt}^{7}$ for a review). The droplets are transported out of their production zone by turbulent diffusion and convective flow, which are counterbalanced by inertial resistance of the particles and gravitational forces. While the sea-spray droplets are airborne, they interact with the scalar fields of temperature and humidity by exchanges of heat and moisture. ${ }^{8}$ These interactions result in the droplets changing size, and thereby their transport properties. Ultimately, sea-spray droplets may evaporate almost completely resulting in sea-salt particles.

The total concentration of sea-spray particles in the atmosphere thus results from freshly produced aerosols, relatively abundant near the surface, and a contribution of aged particles that have undergone various transport and microphysical processes. Due to the many processes involved, the dependence of the aerosol concentration on meteorological parameters is not a simple relation. Generally speaking, wind speed (production and transport) and humidity (evaporation and condensation) are the more prominent parameters that control the sea-spray aerosol concentration. In coastal zones, the aerosol loading of the atmosphere is more complicated due to the presence of many different types of aerosol sources. These sources can be natural (surf and rural aerosols) or due to anthropogenic activities (traffic, urban, industrial aerosols). As a result, the aerosol concentration and composition in the coastal zone are even more complicated functions of meteorological parameters than over open ocean.

In view of the difficulties of obtaining the aerosol extinction directly, the Navy has identified a need for aerosol models that predict the aerosol extinction as a function of (simple) meteorological parameters. An important milestone in predicting the aerosol extinction for open ocean conditions was attained by the development of the Navy Aerosol Model (NAM) by Gathman, ${ }^{9}$ and it represented one of the first efforts to model aerosol extinction solely on the basis of meteorological parameters. The NAM applies a two-step procedure to provide the aerosol extinction at a Naval ship's deck height for open ocean conditions. In the first step, the aerosol concentration is calculated using statistical relations between the concentration and meteorological parameters. This step is followed by a Mie calculation to yield aerosol extinction (and scattering).

\section{THE NAM MODEL}

The original NAM has been updated on the basis of new experimental ${ }^{10,11}$ and theoretical ${ }^{12,13}$ evidence, and it is presently included in the widely used USAF MODTRAN transmission code. ${ }^{14}$ The NAM describes the marine aerosol concentration by three lognormal modes, centered at $0.03,0.24$ and $2.0 \mu \mathrm{m}$ particle radius, respectively. A zero-order mode (also centered at $0.03 \mu \mathrm{m}$ radius) describes the contribution of non-marine aerosols, such as dust particles. The mode amplitudes are related to the meteorological conditions by empirical relations that have been found from a statistical analysis of experimental aerosol data as a function of meteorological parameters.

In the NAM the aerosol distribution is assumed to be representable as a sum over four lognormally distributed modes: ${ }^{9,13}$

$$
\frac{d N}{d r}=\sum_{i=0}^{3} N_{i} f_{i}(r)
$$

where $N_{i}$ is the amplitude, and the shape function $f_{i}(r)$ is a lognormal distribution normalized to unity:

$$
f_{i}(r)=\frac{1}{\sqrt{2 \pi} \sigma_{i} \exp \left(\sigma_{i}^{2} / 2\right) \rho_{i}} \exp \left(-\frac{\left[\ln \left(r / \rho_{i}\right)\right]^{2}}{2 \sigma_{i}^{2}}\right)
$$

For historical reasons the amplitudes for each mode are defined by $\left\{A_{i}\right\}_{i=0, \ldots, 3}$ where $N_{i}=10^{A_{i}}$. Each shape function $f_{i}(r)$ is characterized by the width $\sigma_{i}$ and the center radius $\rho_{i}$. For each mode, $\sigma_{i}=\sqrt{0.5}$ and the 
center radii $\rho_{i}$ are are relative humidity-based corrections to the nominal center radii:

$$
\rho_{i}=R_{i}\left(\frac{G_{i}^{A}-\left(R H_{10} / 100\right)}{G_{i}^{B}\left(1-R H_{10} / 100\right)}\right)^{1 / 3}
$$

where $R H_{10}$ is relative humidity measured at 10 meters above the surface, and $R_{0}=0.03 \mu \mathrm{m} R_{1}=0.03 \mu \mathrm{m}$, $R_{2}=0.24 \mu \mathrm{m}$, and $R_{3}=2.0 \mu \mathrm{m}$. The coefficients $G_{i}^{A}$ and $G_{i}^{B}$ are the Gerber coefficients, and the values for modes $1-3$ can be found in van Eijk. ${ }^{15}$ In fig. 1 the NAM modes $0-3$ are displayed.

It is emphasized that NAM only aims at providing a reasonable estimate of the aerosol extinction at deck height. In view of the complexity of the processes outlined above, it is not realistic to expect a statistical model to yield an exact prediction of the aerosol extinction at an arbitrary point in space and time. For this, a more elaborate mesoscale meteorological model in combination with a world-wide database of aerosol sources would be required. However, the advantage of NAM over a more complex model is that the NAM can be driven with just a few in-situ meteorological parameters and that the NAM requires limited computational power.

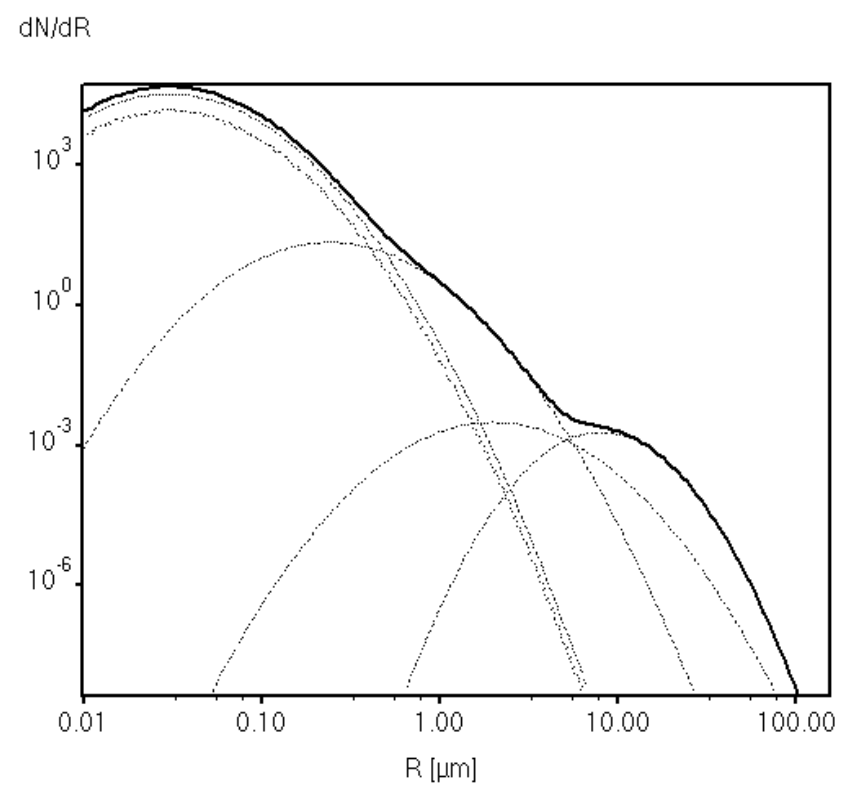

Figure 1. The contribution of each of the five modes to the entire ANAM particle size spectrum. NAM comprises modes $0-3$. Modes 0 and 1 share the same center radius, $0.03 \mu \mathrm{m}$; mode 2 center $=0.24 \mu \mathrm{m}$; and mode 3 center $=2.0 \mu \mathrm{m}$. Mode 4 , centered at $=8.0 \mu \mathrm{m}$, represents the ANAM upgrade.

\section{THE ANAM EXTENSION TO NAM}

Over the years, it has become clear that the NAM code is less successful in coastal regions ${ }^{16}$ and at other heights than deck height $(10 \mathrm{~m}) \cdot{ }^{17}$ The latter shortcoming is partially addressed by the NOVAM model, ${ }^{18}$ presently included in MODTRAN, which extends the NAM aerosol concentration to heights above $10 \mathrm{~m}$. However, in view of the ever-increasing threat of sea-skimming missiles, an urgent need exists for a near-surface version of the NAM. Therefore, the Advanced Navy Aerosol Model (ANAM) is being developed to extend the NAM aerosol concentration from 10 meters down to the wave surface.

The initial development of the ANAM focuses on the behavior of relatively large (radius in excess of 5 $\mu \mathrm{m})$ aerosols. The rationale for this is twofold: first, it has been suggested ${ }^{17}$ that the NAM underestimates the concentration of these larger aerosols resulting in an estimate of the (IR) extinction that is too low. ${ }^{10}$ Secondly, turbulence is less efficient in transporting these heavy aerosols upward from the sea surface, which will (theoretically) result in steeper vertical concentration gradients. ${ }^{19}$ The aim of the ANAM development is to 
extend the NAM by a height-dependent $4^{\text {th }}$ mode, centered at a radius larger than $2 \mu \mathrm{m}$ (nominal radius of $3^{\text {rd }}$ mode in NAM). In a later phase of the development, the necessity of introducing height dependence in the other (NAM) modes will be assessed.

Like its predecessor NAM, the ANAM is being developed by establishing empirical relations between the aerosol concentration and meteorological parameters. ${ }^{20}$ Unfortunately, measurements of vertical aerosol concentrations between the wave surface and deck height are sparse and almost exclusively limited to the Rotorod technique deployed by TNO. ${ }^{21}$ These data sets, which had been acquired in coastal areas and to a smaller extent under open ocean conditions, have been exploited to add a $4^{\text {th }}$ large particle mode to NAM and to develop the ANAM. ${ }^{22}$ A consensus parameterization of the ANAM was obtained, which yielded good results for both data sets. Typically, the ANAM predicts the experimental aerosol concentration to within a factor of 3 . This result was confirmed using an independent Rotorod data set that had not been used in the analysis.

ANAM provides an additional height-dependent mode $^{15}$ for larger particles $(R>5 \mu \mathrm{m})$, denoted the ANAM mode or $4^{\text {th }}$ mode. As with the other modes, the $4^{\text {th }}$ mode is completely characterized by the amplitude $N_{4}$ (defined by the relation $N_{4}=10^{A_{4}}$ ), the width $\sigma_{4}$, and the nominal center radius $R_{4}$. In ANAM version 3.0, $\sigma_{4}=0.5, R_{4}=8.0$, and

$$
A_{4}=-2.5+0.07 U_{10}-0.07 Z
$$

$Z$ denotes height above water surface, and $U_{10}$ denotes the wind speed measured at a 10 meter height. The appearance of the height $Z$ in eqn. 5 generates the vertical height-dependence of the ANAM model. The influence of height on the aerosol concentration will be apparent in the subsequent studies of transmission along two different propagation paths.

It is clear from eqn. (5) that the wind speed will influence the ANAM aerosol distribution, and the influence is modified by height. It is also true that relative humidity influences the distribution due to the change in value of the radius of the $4^{\text {th }}$ mode. The mode dependence is calculated according to eqn. (4). In fig. 1 the four modes used in ANAM are shown. For this version of ANAM (3.0) the Gerber coefficients are defined by: $G_{4}^{A}=G_{3}^{A}$ and $G_{4}^{B}=G_{3}^{B}$.

\section{THIS STUDY}

Preliminary studies by Van Eijk et al. ${ }^{22}$ indicate that the addition of the $4^{\text {th }}$ mode changes aerosol extinction values by about $20 \%$ as compared to NAM calculations. Closer to the surface, this number is even higher due to the height dependence of the $4^{\text {th }}$ mode. However, a more extensive comparison between NAM and ANAM has not yet been made. This paper fills in this gap by presenting a comparison of aerosol extinctions as predicted by NAM and ANAM for propagation paths that descend beneath deck height. It is desirable to make the model comparison by examining a range of meteorological scenarios that occur over regions of Navy interest, so we restrict the test data to a representative sample of marine atmospheric observations assembled by a group from the Naval Surface Warfare Center.

We will quantify the differences between the NAM and ANAM models for specific scenarios. Two data sets, the R384 sample and the R400 sample, were utilized for the study. These two sets were developed by code T44 of the Naval Surface Warfare Center, Dahlgren VA, and their data description states: "The R384 sample includes an equal number of observations (96) from the Baltic Sea, the Yellow Sea, the Gulf of Oman and the Caribbean Sea. It was desireable to represent each area by 8 randomly selected weather samples per month for a total of $8 \times 12 \times 4=384$ samples. The R384 is meant to be a 'World-Wide' environmental sample of areas of common interest." The R384 samples comprise 60 observations from the North Atlantic (Gulf of Finland); 36 observations from the North Atlantic (450 miles west of Ireland); 77 observations from the Yellow Sea; 19 observations from the East China Sea; 96 observations from the mid Arabian Sea (Gulf of Oman); and 96 observations from the Caribbean.

"The R400 sample was co-developed by T44 several years ago to be representative of a typical variety of naval maritime conditions. The 'Random 400' sample was originally put together as a 'world wide weather' data file. The size of this file was selected as a reasonable compromise between computer run time and statistical accuracy. Locations were chosen to represent both areas where aerosol scattering is significant and areas where molecular absorption dominates. The sample is (approximately) uniform with respect to time of day and time of year." 


\section{A COMPARISON TEST}

A comparison between the NAM and ANAM models requires that representative transmission calculations be performed over the full set of meteorological data contained in the R384+R400 data sets. It is important to vary the input parameters of the study to elucidate the effects of certain parameters on the two models. Thus the first requirement is to create a library of extinction coefficients over all of the data in R384+R400. The aerosol extinction calculated by using NAM results in a single extinction coefficient. This calculation is carried out for each of the 784 meteorological records in the combined data sets. The calculation for each of the 784 records is repeated for the ANAM model. Because of the height dependence of ANAM, it is necessary to calculate a representative aerosol extinction value for each 1-meter thick layer from a set of layers extending from the sea surface to a maximum height of 22 meters, and thus each meteorological record

This degree of resolution of the horizontal near-surface atmospheric layers is necessary to discern the impact of ANAM and the height-dependent $4^{\text {th }}$ mode on the paths of interest for IRST applications. In the cases considered below, the path length is 10 kilometers. We consider two paths for this range: a path with a sensor at 8 meters height and a target at 2 meters height, and a second path with a sensor at 20 meters height, with the target still at 2 meters height.

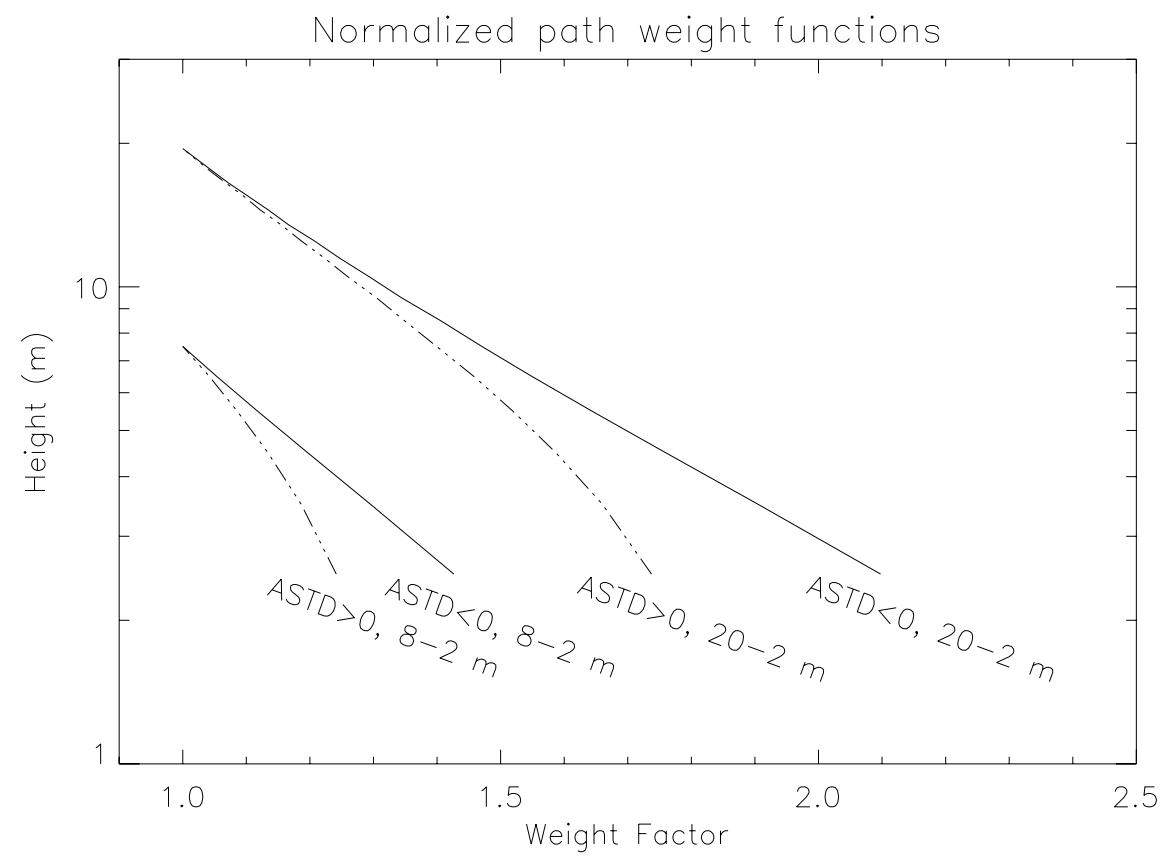

Figure 2. Four normalized path-weighting functions, plotted on a log-linear scale. For the case $T_{\text {air }}-T_{\text {sea }}<0$ and a path from $20 \mathrm{~m}$ to $2 \mathrm{~m}$, the path segment through a $2 \mathrm{~m}$ layer at $2 \mathrm{~m}$ height is more than twice the length of the segment through a $2 \mathrm{~m}$ layer at $20 \mathrm{~m}$ height.

Over a path-length of 10 kilometers, the radius of curvature of the earth becomes a significant factor. In this study the atmosphere is partitioned into concentric shells of equal 1-meter thickness. A geometric straight line between the different heights of the sensor and source is also partitioned into line segments as it cuts through each layer. For our geometry, the longest such segment will be closest to the low end of the path.

The relative segment length is thus a function of the height above the sea surface, and this dependence is described by the path-weighting function. This function is not simply determined by the geometric considerations listed above, since the optical path from sensor to target is not a geometric straight line. Instead there exist refractive gradients in the near-surface environment which can be either positive or negative depending upon the sign of $T_{\text {air }}-T_{\text {sea }}$. For $T_{\text {air }}-T_{\text {sea }}<0$, sub-refractive conditions apply and near-horizontal ray-paths tend to 
curve upward and away from the surface as they propagate. This causes the segments closest to the low end of the path to be even longer relative to the segments at the high end of the path.

Sub-refractive conditions are characterized by a strong vertical refractivity gradient, with a rapid decrease in refractivity as height increases from the surface to a few meters up. The relative sizes of the ray trajectory segments do not change to a great extent for different values of $T_{\text {air }}-T_{\text {sea }}<0$, and for this study we used a single mean weighting function to scale the relative lengths of the differing ray segments. In fig. 2 four weighting functions are displayed. The normalized path-weighting functions are shown for the four different path geometries. There are two cases for ASTD $=T_{\text {air }}-T_{\text {sea }}>0$; the path from $8 \mathrm{~m}$ to $2 \mathrm{~m}$ height, and from $20 \mathrm{~m}$ to $2 \mathrm{~m}$ height. The path is a straight line, and the weighting function shows how the path angle with respect to the local horizontal is decreasing. The second two cases correspond to ASTD $=T_{\text {air }}-T_{\text {sea }}<0$, and the refractive conditions cause rays to bend upward.

The input independent variables upon which the $4^{\text {th }}$ mode of ANAM depends are wind speed, relative humidity, and height. The height variable is implicitly defined by the choice of path as noted above. We will examine the behavior of the R384+R400 sample data at selected levels of both relative humidity and wind speed. Finally, three different wavelengths were examined for the transmission simulations: $\lambda=3.85 \mu \mathrm{m}, \lambda=4.65 \mu \mathrm{m}$, and $\lambda=9.0 \mu \mathrm{m}$.

\section{COMPARISON OF ANAM AND NAM USING METEOROLOGICAL DATA}

In view of the dependencies described in the previous section that are prominent in the ANAM development, the primary input parameters of interest are the relative humidity and wind speed. The differential dependence on wind speed between ANAM and NAM is shown in fig. 3, where the relative transmission computed by the two different aerosol models is compared.

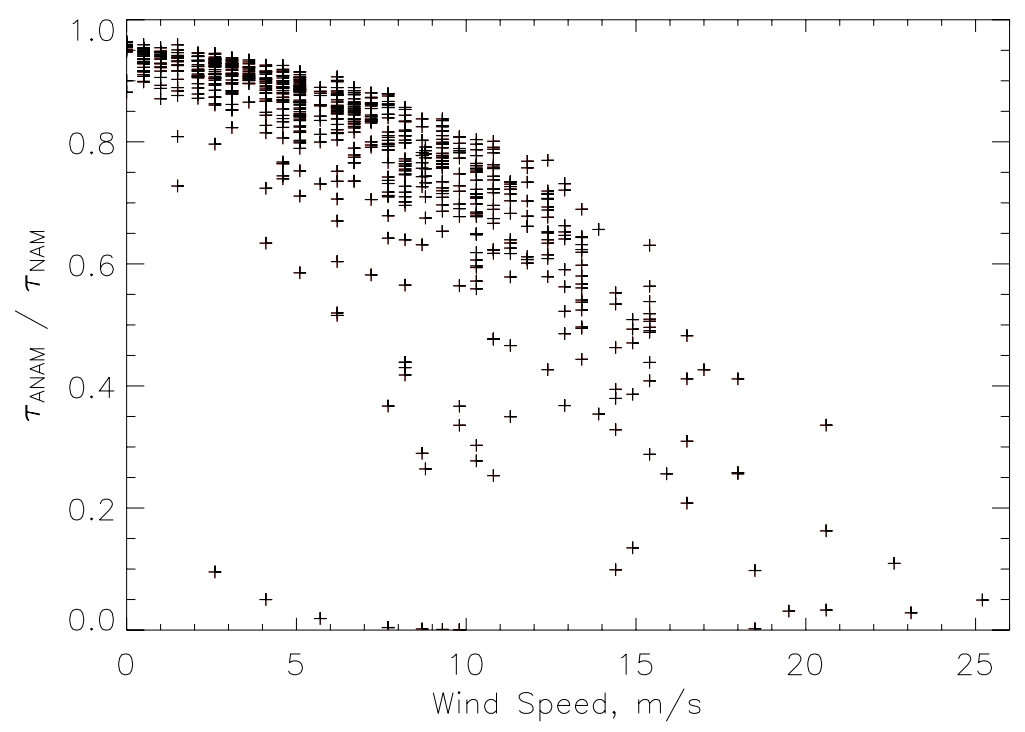

Figure 3. The ratio of transmission at $(\lambda=3.85 \mu \mathrm{m})$ using ANAM to the transmission using NAM for the entire combined set R400+R384, shown as a function of windspeed.

Our analysis of the difference between the two models is based upon a ratio of two different infrared transmission values: a NAM-based value which is the transmission utilizing an aerosol extinction generated by the NAM, and the ANAM-based value which is transmission utilizing an aerosol extinction generated by the ANAM. A raytrace procedure is used to calculate a height(range) functional dependence, so that the relative proportion 
of the path at each level is determined. Transmission is normalized by referencing to the expected free-space value over the same path. The resultant ratio is shown as:

$$
\tau_{\Delta}=\frac{\tau_{\mathrm{ANAM}}}{\tau_{\mathrm{NAM}}}
$$

The factor $\tau_{\Delta}$ indicates the multiplicative factor necessary to adjust the transmission determined using NAM to be equal to the transmission determined by ANAM. Note that $0<\tau_{\Delta}<1$ since the ANAM contains all of the NAM factors and hence incorporates all of the extinction due to NAM, and adds an additional factor due to the $4^{\text {th }}$ mode. Thus in fig. 3 an apparent upper bound for the ratio $\tau_{\Delta}$ as wind speed increases is seen. This is due to the dependence of the $4^{\text {th }}$ mode in ANAM on the wind speed.
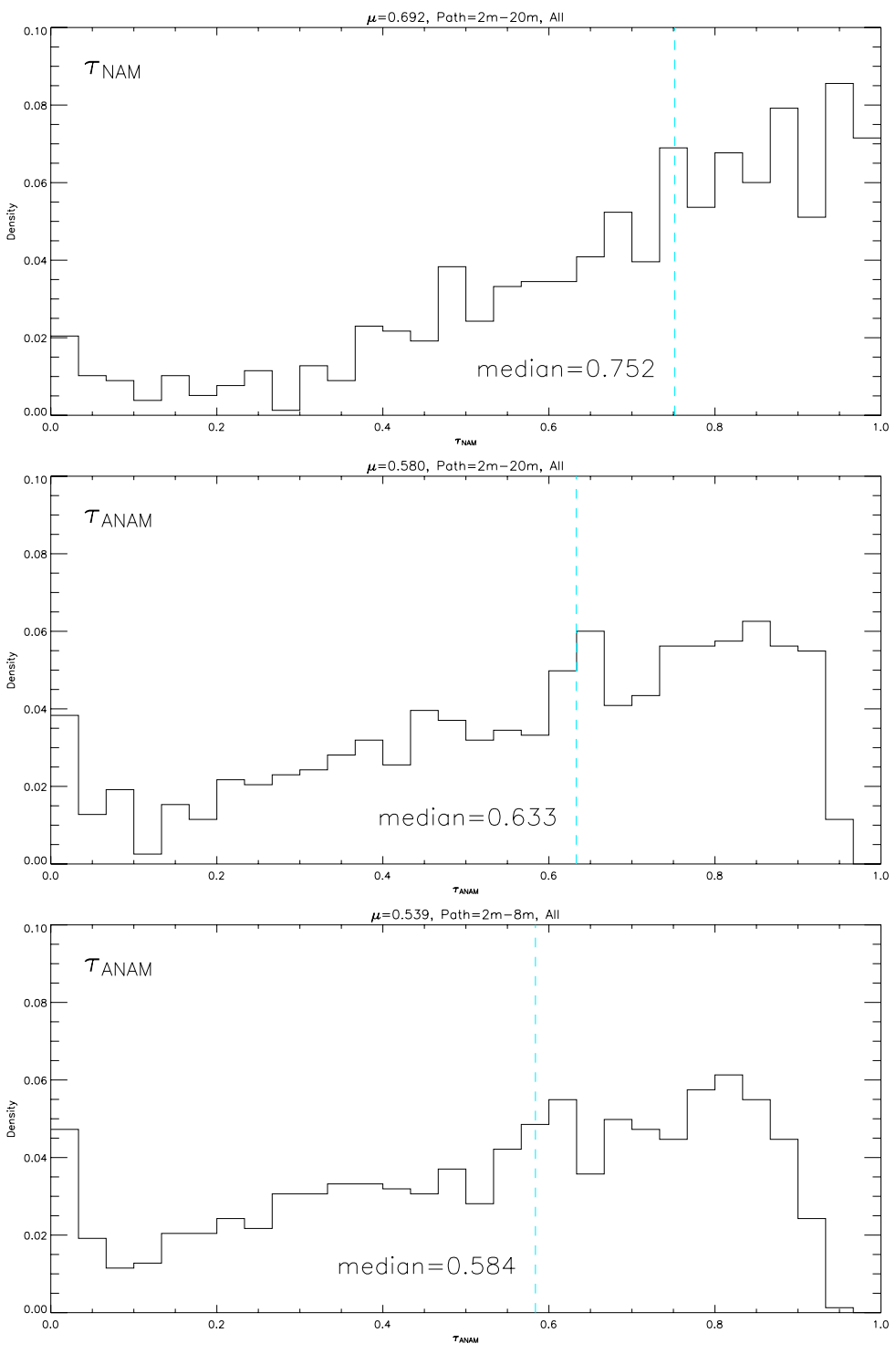

Figure 4. The distribution of normalized transmission values using NAM for the entire combined set R400+R384 is shown in the upper plot over the path starting at $20 \mathrm{~m}$ and ending at $2 \mathrm{~m}$. The similar distribution for transmission using ANAM is shown in the middle plot. The change induced by switching to the path from $8 \mathrm{~m}$ to $2 \mathrm{~m}$ is shown in the bottom plot. Because NAM is height independent the distribution is the same for NAM over the path from $8 \mathrm{~m}$ to $2 \mathrm{~m}$.

A broad comparison of the transmission determined by the NAM and ANAM models is shown in fig. 4. The 
three figures show a distribution of normalized transmission values over the entire combined set R400+R384: 784 meteorological events. The upper plot shows the distribution of normalized transmission values using NAM, and the middle plot shows the same plot when ANAM is utilized as the aerosol model. There are significant differences in the two models even from this broad comparison. A single indicator of the difference between the two can be seen by comparing the median value of 0.752 for the NAM-based distribution to the median 0.633 for the ANAM-based distribution in the middle plot, and finally to the median value 0.584 for the more stressing path from $8 \mathrm{~m}$ to $2 \mathrm{~m}$ in the bottom plot. Note that because the NAM model is height independent, the distribution of transmission values does not change for a switch in paths.
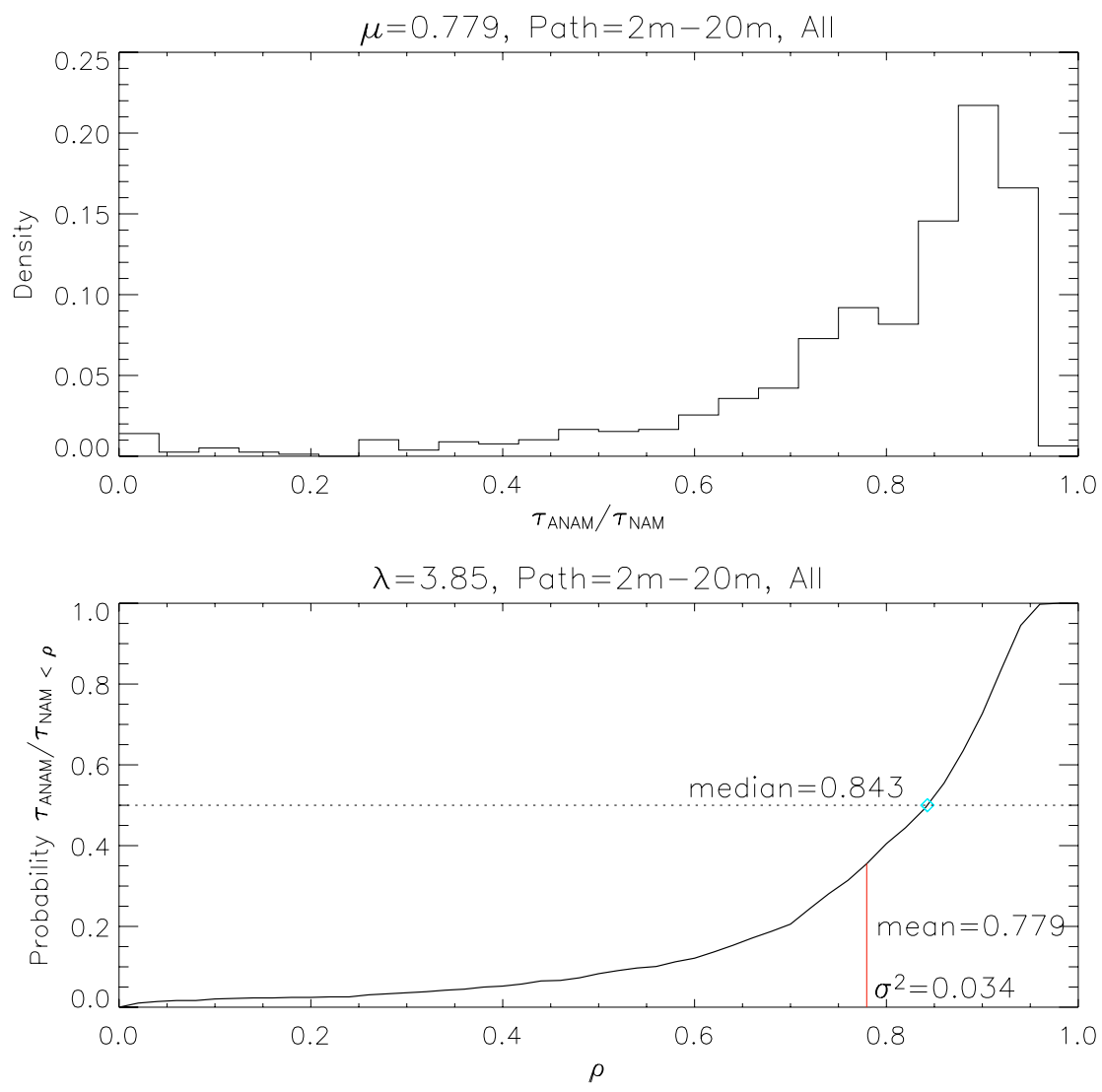

Figure 5. The distribution function for the ratio of transmissions calculated for ANAM over the transmission for NAM, for all meteorological conditions in the database. The path is from a sensor height of $20 \mathrm{~m}$, and a target height of $2 \mathrm{~m}$ at a range of $10 \mathrm{~km}$, and $\lambda=3.85 \mu \mathrm{m}$. This distribution indicates in a rough sense the 'mean' change in transmission due to replacement of the NAM model by the ANAM model.

For the remainder of this paper, the transmission factor $\tau_{\Delta}$ is used to provide the comparative effects of ANAM vs. NAM. A baseline indication of the contrast between NAM and ANAM is shown in fig. 5 in which the full R384+R400 data set is used. The path is from a sensor height of $20 \mathrm{~m}$ and a target height of $2 \mathrm{~m}$ at a range of $10 \mathrm{~km}$. For this calculation, $\lambda=3.85 \mu \mathrm{m}$. Also shown is the calculation of a cumulative probability distribution to determine the probability that the effective transmission factor $\tau_{\Delta}$ is less than a specified ratio $\rho$.

The effect of changing the propagation path from $2 \mathrm{~m}-20 \mathrm{~m}$ to $2 \mathrm{~m}-8 \mathrm{~m}$ is shown in fig. 6 . Thus fig. 5 and fig. 6 provide a reference distribution showing the baseline 'mean' difference in the ANAM and NAM models. In the following figures, we compare the two models in a similar way but with the additional restriction of examining a subset of one of the relevant parameters. 

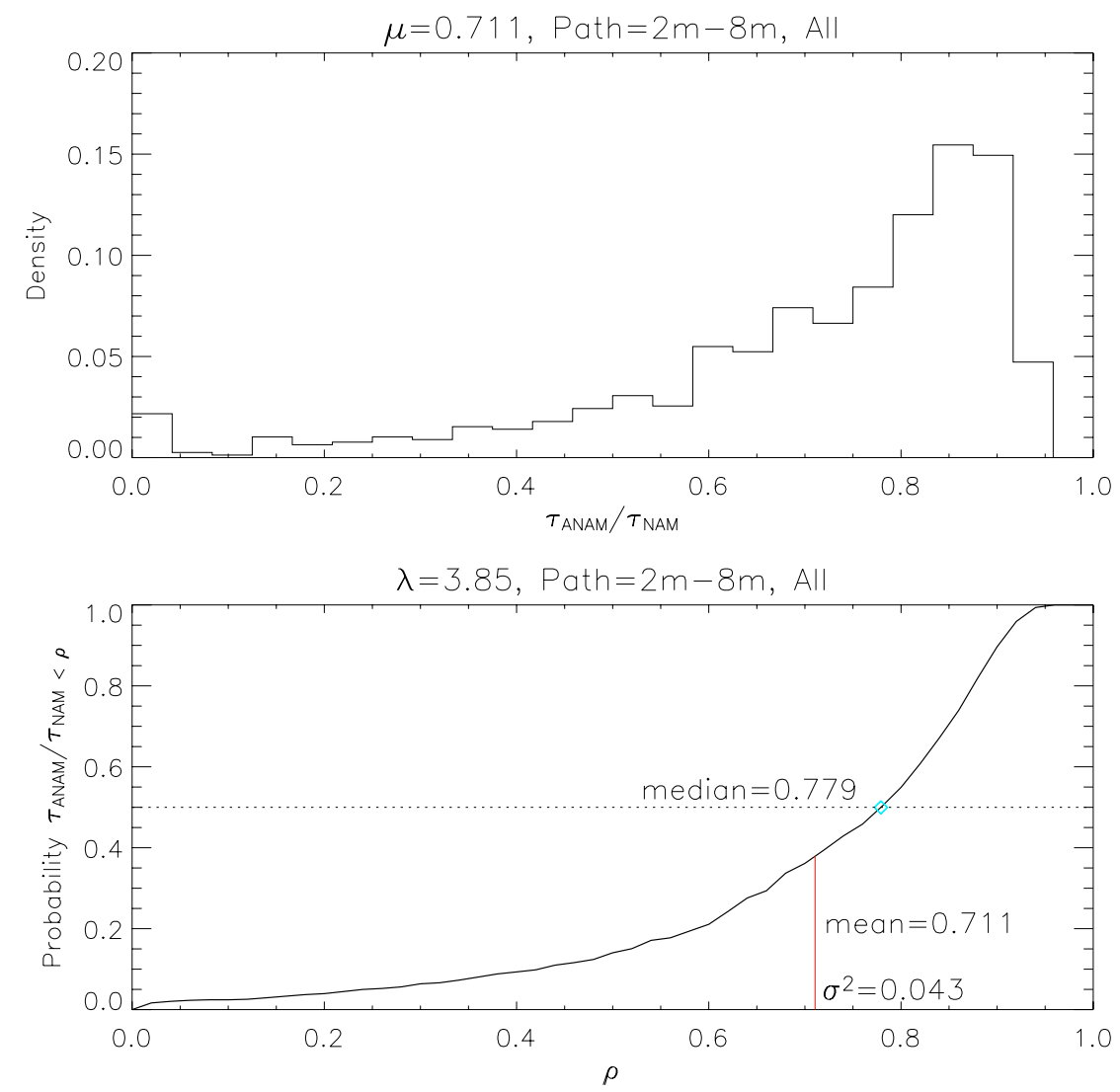

Figure 6. The distribution function for the ratio of transmissions calculated for ANAM over the transmission for NAM, for all meteorological conditions in the database. The path shown here is the more stressing case: a sensor height of $8 \mathrm{~m}$, and a target height of $2 \mathrm{~m}$ at a range of $10 \mathrm{~km}$ with $\lambda=3.85 \mu \mathrm{m}$. This distribution indicates in a rough sense the 'mean' change in transmission for the ANAM model.

\section{COMPARISON OF DISTRIBUTION FUNCTIONS FOR PARAMETER VARIATIONS}

The remainder of this report will present a set of cumulative distribution functions. The event space over which the probability is calculated is the ratio $\tau_{\Delta}$ (defined in eqn. 6) between the ANAM-derived transmission and the NAM-derived transmission. Although significant differences between the two models are apparent in figs. 5, 6 , the more striking contrasts appear when a subset of the full range of meteorological conditions is selected. The subsets are chosen to isolate a 'stressing' end of a parameter range: $50^{\text {th }}$ and $90^{\text {th }}$ percentiles of wind speed or relative humidity.

The effect of wind-speed on the ratio $\tau_{\Delta}$ (eq. 6) is shown in fig. 7 for the $2 \mathrm{~m}-20 \mathrm{~m}$ path and in fig. 8 for the $2 \mathrm{~m}-8 \mathrm{~m}$ path. The bottom panel of fig. 8 is striking: for the $90 \%$ stressing wind-speeds (in this case, $>11 \mathrm{~m}$ $\mathrm{sec}^{-1}$ ), the median value for $\tau_{\Delta}$ is 0.39 . This is strong testimony to the modifications in ANAM to correct the NAM underestimation of large aerosol particles. These large particles are described by the $4^{\text {th }}$ mode, and as shown in eqn. 5 the mode amplitude is a function of both height and wind-speed.

\section{SUMMARY AND CONCLUSIONS}

We calculated statistics similar to the results displayed in figs. 7 and 8 for different parameter variations, and for different wavelengths. In table 1 we show the results of these calculations for all combinations of parameter 

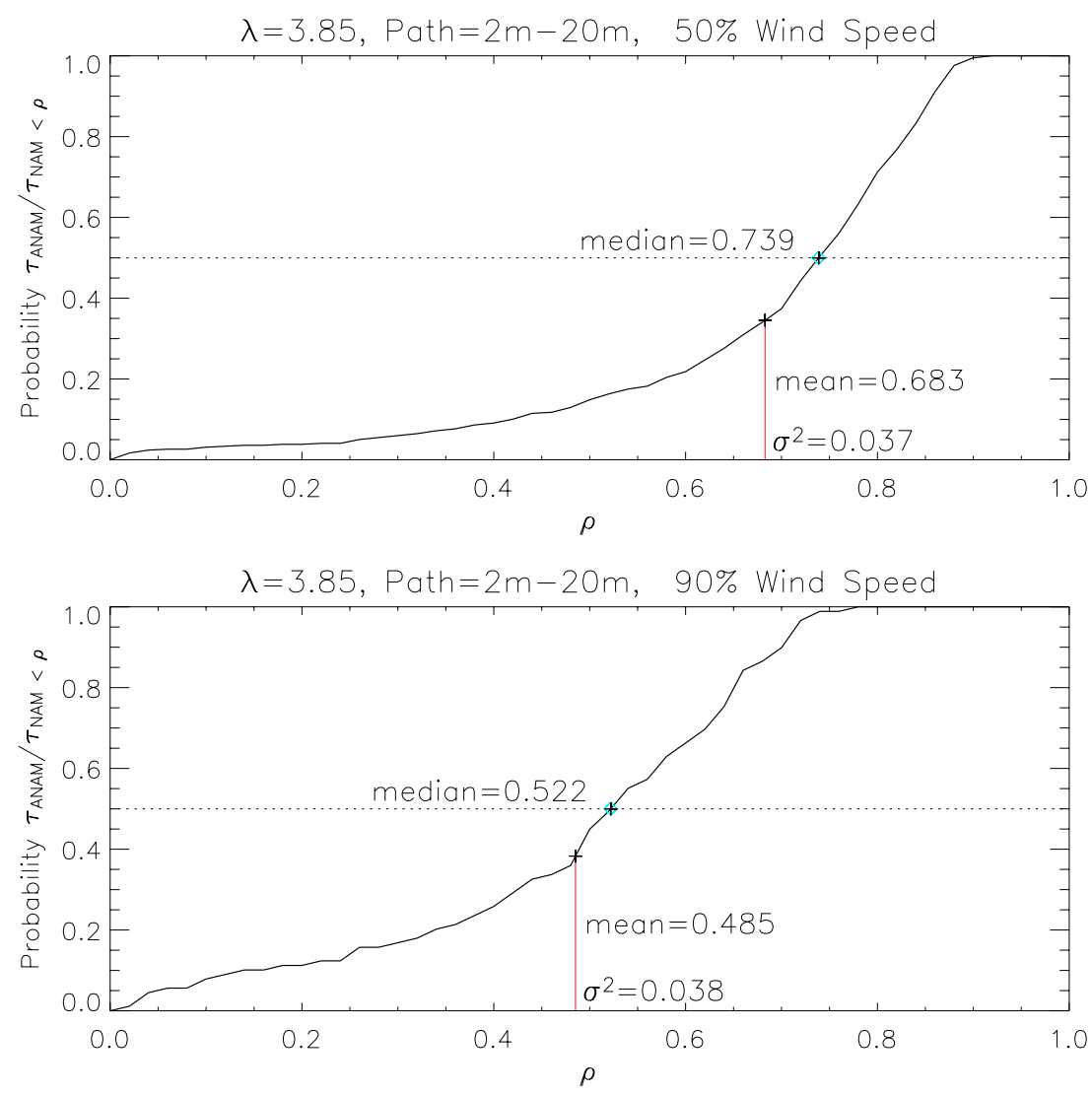

Figure 7. The path is from a sensor height of $20 \mathrm{~m}$, and a target height of $2 \mathrm{~m}$ at a range of $10 \mathrm{~km}$, and $\lambda=3.85 \mu \mathrm{m}$. The distribution is calculated for conditions of $50^{\text {th }}$ percentile wind speed and $90^{\text {th }}$ percentile wind speed.

settings: wavelengths of $3.85 \mu \mathrm{m}, 4.65 \mu \mathrm{m}$, and $9.0 \mu \mathrm{m} ; 2 \mathrm{~m}-20 \mathrm{~m}$ path vs. $2 \mathrm{~m}-8 \mathrm{~m}$ path; $50 \%$ vs. $90 \%$ humidity, and $50 \%$ vs. $90 \%$ wind-speed.

In the figures, both mean and median values for the distributions are shown. The median is a more conservative and arguably more reliable figure of merit for the comparison. One could argue that a model upgrade that results in a change by a factor of two is significant, and warrants a migration to the newer model. The $90^{\text {th }}$ percentiles of both the wind speed and relative humidity have median values less than or equal to 0.54 in all cases considered, and for the low path and a wavelength of $9.0 \mu \mathrm{m}$, the median $=0.34$ is a factor of three difference between the two models.

The goal of this study is to demonstrate the significance of the difference between using ANAM vs. NAM as the aerosol extinction model. For stressing atmospheric conditions, or in the case of a largely near surface path, an upgrade from the NAM to the ANAM model for a more accurate calculation of the extinction due to aerosol is recommended.

\section{ACKNOWLEDGMENTS}

This work was supported by Dr. R. Ferek and Mr. J. Buss of the Office of Naval Research. The development of ANAM is jointly sponsored by the Office of Naval Research and the Royal Netherlands Navy. The TNO Rotorod data has been acquired with financial support of the Royal Netherlands Navy, the European Union and the Office of Naval Research through various assignments. 

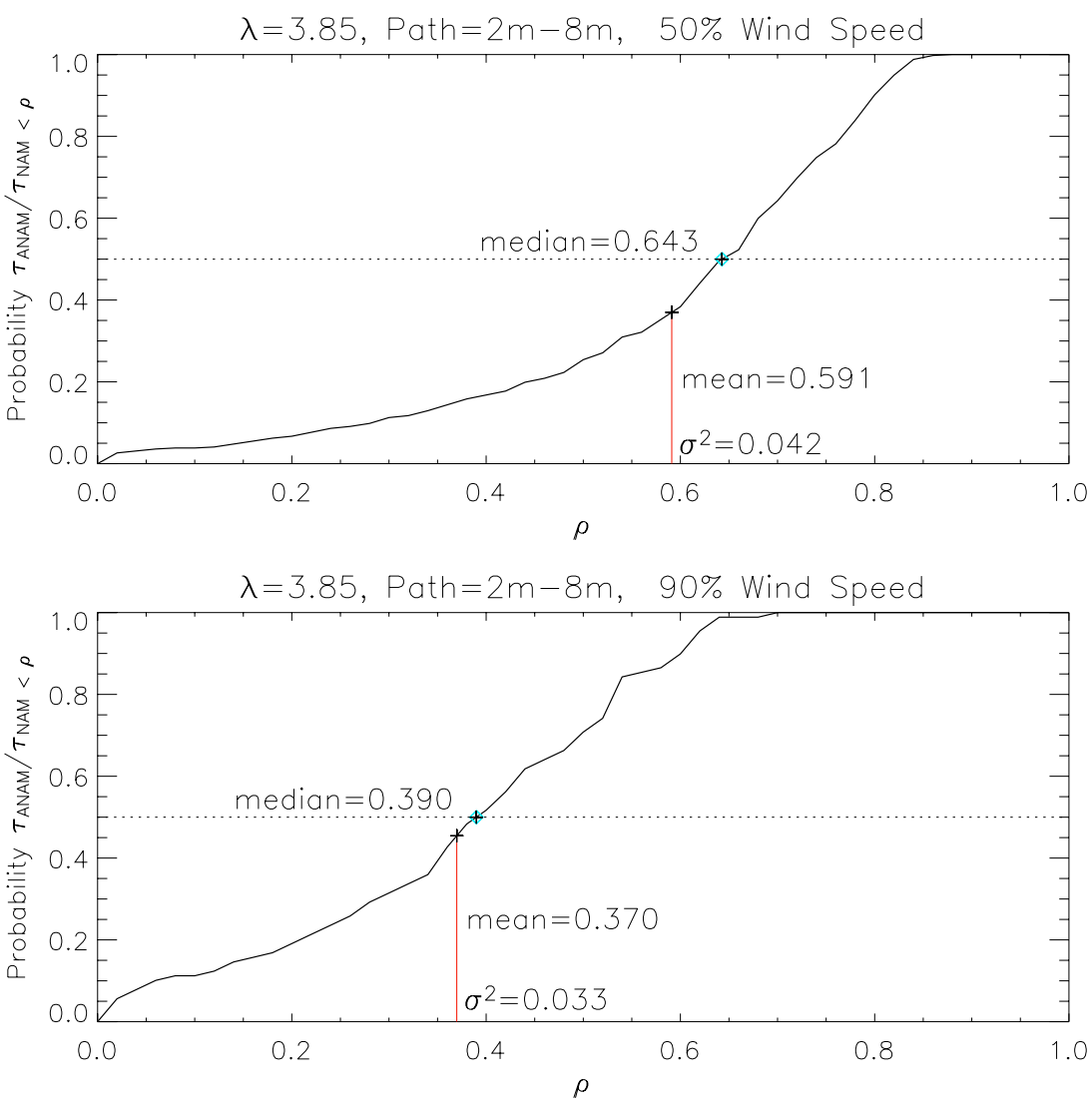

Figure 8. The path is from a sensor height of $8 \mathrm{~m}$, and a target height of $2 \mathrm{~m}$ at a range of $10 \mathrm{~km}$, and $\lambda=3.85 \mu \mathrm{m}$. The distribution is calculated for conditions of $50^{\text {th }}$ percentile wind speed and $90^{\text {th }}$ percentile wind speed.

\section{REFERENCES}

1. Blanchard, D.C., "The electrification of the atmosphere by particles from bubbles in the sea", Prog. Oceanogr., 1, 71-202, 1963.

2. Spiel, D.E., "The sizes of jet drops produced by air bubbles bursting on sea- and fresh-water surfaces", Tellus, 46B, 325-338, 1994.

3. Spiel, D.E., "A hypothesis concerning the peak in film drop production as a function of bubble size", J. Geophys. Res., 102, 1153-1161, 1997.

4. Monahan, E.C., D.E. Spiel and K.L. Davidson, "A model of marine aerosol generation via whitecaps and wave disruption", in Oceanic Whitecaps, edited by E.C. Monahan and G. McNiocaill, pp. 167-174, D. Reidel, Norwell, Mass., 1986.

5. Andreas, E.L., "A new sea spray generation function for wind speeds up to $32 \mathrm{~m} / \mathrm{s}$ ", J. Phys. Oceanogr., 28, 2175-2184, 1998.

6. Miles, J.W., "On the generation of surface waves by shear flow", J. Fluid Mech., 3, 185-204, 1957.

7. Belcher, S.E., and J.C.R. Hunt, "Turbulent flow over hills and waves", Annu. Rev. Fluid. Mech., 30, 507-538, 1998.

8. Pruppacher, H.R. and J.D. Klett, "Microphysics of clouds and precipitation", Reidel Publishers, Dordrecht, The Netherlands, 1978.

9. Gathman, S. G., "Optical Properties of the marine aerosol as predicted by the Navy Aerosol Model," Opt. Eng. 22, 57-62, 1983.

10. G. DeLeeuw, G., S. Parashar, P.M. Park, S.J. Perry, P.L. Roney and M.H. Smith. "Aerosol study in the North Atlantic 1983", NATO AC/243 (Panel 4/RSG8) Report 1986-01, 1986. 
$2 \mathrm{~m}-20 \mathrm{~m}$ path

\begin{tabular}{|l|c|c|c|}
\hline \hline \multirow{3}{*}{$50 \%$ humidity } & $\lambda=3.85 \mu \mathrm{m}$ & $\lambda=4.65 \mu \mathrm{m}$ & $\lambda=9.0 \mu \mathrm{m}$ \\
\cline { 2 - 4 } & median=0.77 & median=0.77 & median=0.75 \\
& $\mu=0.71$ & $\mu=0.71$ & $\mu=0.69$ \\
\hline $90 \%$ humidity & median=0.54 & median=0.54 & median=0.52 \\
& $\mu=0.49$ & $\mu=0.48$ & $\mu=0.47$ \\
\hline $50 \%$ wind & median=0.74 & median=0.74 & median=0.72 \\
& $\mu=0.68$ & $\mu=0.68$ & $\mu=0.66$ \\
\hline $90 \%$ wind & median=0.52 & median=0.52 & median=0.49 \\
& $\mu=0.49$ & $\mu=0.48$ & $\mu=0.46$ \\
\hline \hline \multirow{2}{*}{$50 \%$ humidity } & $\lambda=3.85 \mu \mathrm{m}$ & $\lambda=4.65 \mu \mathrm{m}$ & $\lambda=9.0 \mu \mathrm{m}$ \\
\cline { 2 - 4 } & median=0.68 & median=0.68 & median=0.66 \\
& $\mu=0.63$ & $\mu=0.63$ & $\mu=0.61$ \\
\hline $90 \%$ humidity & median=0.42 & median=0.42 & median=0.38 \\
& $\mu=0.39$ & $\mu=0.39$ & $\mu=0.37$ \\
\hline $50 \%$ wind & median=0.64 & median=0.64 & median=0.62 \\
& $\mu=0.59$ & $\mu=0.59$ & $\mu=0.57$ \\
\hline $90 \%$ wind & median=0.39 & median=0.39 & median=0.35 \\
& $\mu=0.37$ & $\mu=0.37$ & $\mu=0.34$ \\
\hline
\end{tabular}

Table 1. The transmission factor $\tau_{\Delta}$ computed for the meteorological data in the R400+R384 database, subject to the restrictions indicated by the table entries. The median and the mean $\mu$ are reported for the restricted set of $\tau_{\Delta}$ in each cell.

11. Hughes, H.G., "Evaluation of the LOWTRAN-6 Navy maritime aerosol model using 8 to $12 \mu \mathrm{m}$ sky radiances", Opt. Eng. 26, 1155-1160, 1987.

12. Gerber, H.E., "Relative humidity parameterization of the Navy Aerosol Model (NAM)", NRL report 8956, NRL Washington DC, 1985.

13. Gathman, S. G., "A preliminary description of NOVAM, the Navy Oceanic Vertical Aerosol Model," NRL report 9200, NRL Washington DC, 1989.

14. Kneizys, F.X., L.W. Abreu, G.P. Anderson, J.H. Chetwynd, E.P. Shettle, A. Berk, L.S. Bernstein, D.C. Robertson, P. Acharya, L.S. Rothman, J.E.A. Selby, W.O. Gallery and S.A. Clough, The Modtran 2/3 report and Lowtran 7 model, Philips Laboratory PL/GPOS, Hanscom AFB, MA, 1996.

15. van Eijk, A. M. J., "The ANAM-3.0 development," TNO (the Netherlands Organisation for Applied Scientific Research) report FEL-01-C187, 2001.

16. A.M.J. Van Eijk and G. DeLeeuw (1992), "Modeling aerosol extinction in a coastal environment", SPIE Proc. 1688, 28-36, 1992.

17. G. DeLeeuw, K.L. Davidson, S.G. Gathman and R.V. Noonkester, "Modeling of aerosols in the marine mixed-layer", SPIE Proc. 1115, 27-1 to 27-8, 1989.

18. S.G. Gathman, G. DeLeeuw, K.L. Davidson and D.R. Jensen, "The Navy Oceanic Vertical Aerosol Model: progress report", Proc. AGARD 454 paper 17, 1989.

19. C.W. Fairall, K.L. Davidson and G.E. Schacher, "Meteorological models for optical properties in the marine atmospheric boundary layer", Opt. Eng. 21, 847-857, 1982.

20. S.G. Gathman, A.M.J. van Eijk and L.H. Cohen, "Characterizing large aerosols in the lowest levels of the marine atmosphere," SPIE Proc. 3433, 41-52, 1998.

21. DeLeeuw, G., "Vertical profiles of giant particles close above the sea surface", Tellus 38B, 51-61, 1986.

22. A.M.J. van Eijk, L.H. Cohen, L.J. Navarro and G. De Leeuw, "Near-surface aerosol transmission in the marine environment", SPIE Proceedings 4884, (2002) 160-169. 